\title{
GESTÃO DA INOVAÇÃO EM EMPRESA PAULISTA DO SETOR DE EQUIPAMENTOS MÉDICOS, HOSPITALARES E ODONTOLÓGICOS: EVIDÊNCIAS DE UM ESTUDO DE CASO
}

\author{
RESENDE, Jordão ${ }^{1}$ \\ GOMES, Erasmo ${ }^{1}$ \\ SILVEIRA, Marco Antonio ${ }^{2}$
}

Recebido em: 2014.10.13

Aprovado em: 2015.10.13

ISSUE DOI: $10.3738 / 1982.2278 .1403$

\begin{abstract}
RESUMO: O objetivo deste trabalho é verificar o uso de ferramentas de gestão da inovação em uma pequena empresa produtora de Equipamentos Médicos, Hospitalares e Odontológicos (EMHO) em Ribeirão Preto (SP, Brasil). O modelo utilizado como base teórica para caracterizar as práticas e técnicas de gestão da inovação foi o TEMAGUIDE (COTEC, 1999), o qual propõe 18 ferramentas sobre o assunto. Foi verificado que apesar de algumas restrições típicas do setor e de uma empresa de pequeno porte, há ainda expressivo potencial para implementação e aplicação dessas ferramentas.
\end{abstract}

Palavras-chave: Gestão da inovação. Gestão tecnológica. Equipamentos médicos, odontológicos e hospitalares.

SUMMARY: The aim of this study is to verify the use of tools for managing innovation in a small-producer of Medical Hospital and Dental Equipment (EMHO) in Ribeirão Preto (SP, Brazil). The model used as the theoretical basis for characterizing the practices and techniques of innovation management was TEMAGUIDE (COTEC, 1999), which offers 18 tools on the subject. It was found that although some restrictions typical of the industry and a small business, there is still significant potential for implementation and application of these tools.

Keywords: Innovation management. Technology management. Medical and dental instruments

\section{INTRODUÇÃO}

A quebra de barreiras geográficas, aliadas à velocidade na produção e na transmissão de informações, indica que se vive um momento de mudanças aceleradas e constantes, em várias áreas, especialmente no campo tecnológico. O rápido desenvolvimento da sociedade e dos mercados nas últimas décadas implica às empresas um mercado repleto de desafios e competitividade. A medida da competitividade de uma empresa é a capacidade de transformar seu setor para que gere nova riqueza, isto é, depende de sua capacidade de gerar mudanças. Desse modo a inovação é essencial para a empresa manter a competitividade (LIMAS et al., 2008).

A gestão tecnológica e de inovação é atualmente uma prática essencial para qualquer negócio, principalmente para aqueles inseridos em mercados que exigem alto grau de inovação para manter a competitividade - como é o caso, por exemplo, do setor de equipamentos, médicos, hospitalares e odontológicos -, auxiliando as empresas a administrar as operações existentes de forma mais eficaz e a desenvolverem-se estrategicamente para fortalecer seus recursos, seu know-how e suas competências.

As ferramentas de gestão da inovação são apresentadas como um conjunto de técnicas e práticas de apoio a gestão da inovação e tornam-se o foco do tema abordado para a realização deste trabalho.

\footnotetext{
${ }^{1}$ Faculdade de Economia, Administração e Contabilidade de Ribeirão Preto. FEARP-Univ.de São Paulo - USP

${ }^{2}$ Centro de Tecnologia da Informação Renato Archer
} 
Existem várias propostas cujo objetivo é organizar e sistematizar estas práticas e técnicas, porém, para o presente trabalho, optou-se pela utilização do TEMAGUIDE (COTEC, 1999). Além da conceituação básica sobre gestão da inovação e suas ferramentas, este trabalho apresenta também a caracterização do setor de equipamentos médicos, hospitalares e odontológicos (EMHO), a metodologia de desenvolvimento, com foco na pesquisa de campo; e as análises dos dados coletados, com as conclusões. O interesse pelo setor de EMHO é decorre da característica inovadora atribuída às suas empresas e do seu destaque no Estado de São Paulo.

O objetivo geral do trabalho é, portanto, identificar, por meio da realização de um estudo de caso, ferramentas, e respectivas técnicas específicas, de gestão da inovação adotadas em uma empresa produtora de equipamentos médicos, hospitalares e odontológicos (EMHO) localizada no Estado de São Paulo, em especial, no município de Ribeirão Preto. O referencial utilizado para tanto será aquele constante em Pautas Metodologicas em Gestión de la Tecnologia y de la Innovación para Empresas - TEMAGUIDE (COTEC, 1999). Como objetivos específicos tem-se: i) mapear as práticas conhecidas e utilizadas pelasempresas selecionadas, tendo como base o conjunto de práticas apresentadas no TEMAGUIDE (COTEC, 1999); ii) avaliar as práticas de apoio à gestão da inovação aplicáveis aos processos-chave da empresa selecionada; iii) identificar um conjunto adequado de práticas que melhor se adequem às empresas selecionadas. Espera-se com o presente trabalho contribuir para a ampliação do entendimento sobre a situação atual relacionada ao conhecimento, domínio e aplicação, por empresas do setor de equipamentos médicos, hospitalares e odontológicos (EMHO), de ferramentas, técnicas específicas e práticas de gestão tecnológica e de inovação, conforme prescrito em TEMAGUIDE (COTEC, 1999), assim como identificar e explorar os limites e potencialidade da adoção dessas práticas em pequenas e médias empresas do setor analisado.

\section{PROCEDIMENTOS METODOLÓGICOS}

Utilizando a classificação proposta por Gil (2002, p.41), a presente pesquisa pode ser caracterizada quanto à abordagem do problema, como qualitativa e quanto aos seus objetivos, como descritiva. Por sua vez, quanto aos procedimentos técnicos utilizados para obtenção dos dados, segundo a classificação de Lakatos e Marconi (2003, p.43), trata-se de pesquisa bibliográfica (ou de fontes secundárias) e pesquisa de campo (ou de fontes primárias). De todo modo, ainda segundo Lakatos e Marconi (2003, p. 186), a realização de pesquisa de campo é indissociável da realização prévia de pesquisa bibliográfica. Assim, a primeira fase do estudo, de caráter descritivo e exploratório, consistiu em revisão bibliográfica sobre gestão da inovação. A segunda fase, também exploratória, consistiu na realização de uma pesquisa de campo realizada junto uma empresa do setor de fabricação de equipamentos médicos, hospitalares e odontológicos (EMHO). Este setor foi escolhido por ser intensivo em desenvolvimento tecnológico e inovação, possuir atividade exportadora e sofrer uma forte concorrência de competidores internacionais. A seleção da empresa, objeto da pesquisa de campo, teve como ponto de partida uma lista de empresas pertencentes ao setor de EMHO, localizadas no município de Ribeirão Preto (SP). O critério final baseou-se na concordância da empresa em participar do estudo em tela. A identificação da empresa será mantida em sigilo, por solicitação da mesma.

Quanto aos procedimentos utilizados para a realização da coleta de dados, no âmbito da pesquisa de campo, utilizou-se de entrevista. Quanto ao tipo, foi empregada entrevista padronizada ou estruturada, na qual "o entrevistador segue um roteiro previamente estabelecido; as perguntas feitas ao indivíduo são

Nucleus,v.12,n.2,out.2015 
predeterminadas. Ela se realiza de acordo com um formulário elaborado e é efetuada de preferência com pessoas selecionadas de acordo com um plano" (LAKATOS; MARCONI, 2003, p. 197).

$O$ roteiro ou formulário de entrevista foi estruturado em torno de catorze questões, sendo cinco abertas e nove fechadas ou dicotômicas. Em caso de resposta afirmativa, o entrevistado era remetido a perguntas de estimação ou avaliação, com campo para justificativa e comentários. Segundo Lakatos e Marconi (2003, p. 206), perguntas de estimação ou avaliação "consistem em emitir um julgamento através de uma escala com vários graus de intensidade para um mesmo item. As respostas sugeridas são quantitativas e indicam um grau de intensidade crescente ou decrescente". O respondente foi o gerente de inovação da empresa, cujo cargo havia sido recém-criado à época da aplicação do questionário.

Com o intuito de homogeneização terminológica e conceitual, e sobre as técnicas e ferramentas de gestão da inovação propostas por TEMAGUIDE (COTEC, 1999) e aquelas aplicadas ou conhecidas pela empresa, foram realizadas cinco sessões de apresentação das ferramentas, assim como, a entrega de um manual sintético ao respondente da empresa. Nessas seções foram esclarecidos os objetivos e formas de aplicação das 18 ferramentas de gestão da inovação propostas por TEMAGUIDE. As entrevistas foram realizadas após as apresentações das ferramentas de gestão da inovação, divididas em duas sessões e auxiliadas por um roteiro de questões e pelo manual sintético de ferramentas de gestão da inovação ambos utilizados pelo pesquisador e pelo entrevistado. Além disso, uma folha de respostas e um gravador foram utilizados apenas pelo pesquisador para auxiliar nos registros das informações. Por fim, foram tabulados os resultados da realização da pesquisa de campo e realizadas as análises dos dados apresentadas na seção referente a resultados.

\section{GESTÃO DA INOVAÇÃO}

Segundo Gavira et al. (2007, p. 80), gestão da inovação envolve “[...] um conjunto de práticas, conceitos e ferramentas que ajuda o tomador de decisão a organizar o processo de geração de inovações, renovação da empresa, geração de novos negócios e de valor em cima de inovação". Ou ainda, [...] a ideia da gestão da inovação é estruturar, sob uma perspectiva estratégica, rotinas e ferramentas na empresa com periodicidade e sistematização, de modo que a inovação não seja algo espontâneo e desarticulado na empresa (FUCCK; VILHA, 2011, p. 14).

De acordo com Saenz e Capote ${ }^{3}(2002$ apud NATUME; CARVALHO; FRANCISCO, 2008, p.3), a gestão tecnológica pode ser definida como:

[...] a gerência sistemática de todas as atividades no interior da empresa com relação à geração, aquisição, início da produção, aperfeiçoamento, assimilação e comercialização das tecnologias requeridas pela empresa, incluindo a cooperação e alianças com outras instituições; abrange também o desenho, promoção e administração de práticas e ferramentas para a captação e/ou produção de informação que permita a melhoria continuada e sistemática da qualidade e da produtividade.

Para Coral et al. (2008) a gestão da inovação, entre outros aspectos, trata da organização e direcionamento de recursos humanos e financeiros com o propósito de aumentar a geração de novos conhecimentos, a geração de ideias de novos produtos e processos e a melhoria dos já existentes. Assim sendo, foram desenvolvidos métodos de gestão que agregam tanto as características objetivas (planejamento, metas, indicadores) como subjetivas (criatividade, cooperação) da inovação tornando-a um

${ }^{3}$ SÁENZ, T.W.; CAPOTE, E.G. Ciência, inovação e gestão tecnológica. Brasília: ABIPTI/SENAI. 2002. 136 p. 
conceito menos dependente de sorte ou da criatividade de alguns e passível de gerenciamento e de estímulo dentro de uma organização. Referindo-se aos desafios da gestão da inovação, Paladino (2010, p. 12) menciona que

O caldo de cultura da inovação requer ingredientes organizacionais diversos, tais como: imaginação e criatividade; liberdade de expressão; adesão dos colaboradores aos objetivos estratégicos das empresas; mix de conhecimentos técnicos, científicos, tácitos e tradicionais; comunicação organizacional eficiente; interdisciplinaridade nas soluções e profunda intimidade com o mercado consumidor. Alguns desses elementos não são domesticáveis ou são de difícil normalização. Diferentemente dos bons manuais da qualidade e produtividade (PALADINO, 2010, p. 12)

Para Quadros e Vilha ${ }^{4}$ (2006 apud FUCK; VILHA, 2011, p. 14),

[...] não há um blue print para gerar inovações, nem para gerir o processo. As demandas/necessidades do gestor da inovação são muito diferenciadas para empresas de setores diferentes e tamanhos diferentes, isto é, cada empresa deve customizar a gestão de seus processos inovativos segundo suas prioridades e recursos.

As inovações não são fruto de geração espontânea, nem criadas no vácuo: são antes de tudo resultado de interações deliberadas e geradas num ambiente propício, num terreno fértil onde as ideias prosperam. Há organizações que se caracterizam por promoverem a inovação, seja de produto, de processo, de serviço, de negócio ou organizacional (SCHERER ; CARLOMAGNO, 2009, p. 22).

\subsection{Importância da gestão da inovação para a competitividade das empresas}

A importância da gestão tecnológica e da inovação é dificilmente questionada, porém muitas vezes ela não é percebida dentro da organização. O fato da gestão tecnológica e da inovação estar diretamente ligada à produção diária de uma empresa faz com que muitas vezes sua importância não seja observada nitidamente no processo de inovação(NATUME; CARVALHO; FRANCISCO, 2008).No entanto, esta situação pode ser revertida através de um olhar mais atento. Autores como Terra, Kruglianskas e Zilbar(1993 apud NATUME; CARVALHO; FRANCISCO, 2008) afirmam que é por meio da gestão tecnológica e da inovação nos diversos processos produtivos da empresa que os desvios, involuntários ou provocados, podem ser observados mediante de práticas diárias que conduzem ao processo de inovação na empresa.

A gestão tecnológica e da inovação auxilia a organização a preparar-se para o futuro, reduzir os riscos comerciais e as incertezas, aumentando sua flexibilidade e capacidade de resposta, sendo a inovação é um fator essencial em todas essas tarefas. A inovação melhora a eficiência e também pode mudar, de forma significativa, os produtos e os processos, o que coloca aempresa em um maior nível de competitividade e permite entrar em novas áreas de negócios (COTEC, 1999, p. 11).

\footnotetext{
${ }^{4}$ VILHA, A.M.; QUADROS, R. Gestão da inovação sob a perspectiva do desenvolvimento sustentável: lições das estratégias e práticas na indústria de higiene pessoal, perfumaria e cosméticos. RAI - Revista de Administração e Inovação, v. 9, n. 3, p. 28-52, 2012.

${ }^{5}$ TERRA, J. C.; KRUGLIANSKAS, I.; ZILBER, S. N. Avaliação da gestão tecnológica na pequena empresa. In: CONGRESSO LATINO AMERICANO DE ENGENHARIA INDUSTRIAL.1., 1993. Anais... Florianópolis: UFSC,1993.
}

Nucleus,v.12,n.2,out.2015 


\subsection{Gestão da inovação em empresas de pequeno porte}

Segundo Perini ${ }^{6}$ et al. ( 2002, apud LIMAS; SCANDELARI; FRANCISCO, 2008) várias ferramentas de gestão tecnológica e da inovação já fazem parte do dia-a-dia das pequenas e médias empresas (PMEs). No entanto, a maioria delas faz parte de programas de melhoria de qualidade ou para resolver necessidades específicas. Além disso, muitas poderiam ser de grande valia, mas por não possuirem difusão adequada, não são aplicadas, sendo necessário um trabalho de capacitação das empresas para sua utilização. Segundo Souza (2003, p. 33), o emprego de ferramentas de gestão tecnológica e da inovação não é disseminado de forma sistemática no ambiente das PMEs, conforme demonstram várias pesquisas realizadas, em especial, no Brasil (MORAIS ${ }^{7}, 1999$ apud SOUZA, 2003).

Souza (2003) aponta ainda para uma importante dificuldade de utilização das ferramentas de gestão tecnológica e da inovação pelas PMEs: "o custo de muitos sistemas e a implementação de estruturas internas para a gestão tecnológica, separam, naturalmente, as chamadas grandes das pequenas empresas, levando-se apenas em conta a variável custo". Os resultados do estudo desenvolvido por Souza (2003, p. 100), em 176 PMEs da região metropolitana de Curitiba, não foram diferentes: “[...] nem uma das ferramentas de gestão tecnológica e da inovação é utilizada formalmente de forma majoritária pelas empresas amostradas [...]". O estudo desse autor indica também que as poucas ferramentas de gestão tecnológica e da inovação que são utilizadas são motivadas por pressões regulatórias, redução do custo do processo e proteção de ativos, e não nas atitudes de gestão. Ainda segundo Souza (2003), há evidências de desalinhamento entre as melhores práticas de gestão tecnológica e de inovação em economias mais desenvolvidas com as das realizadas pelas PMEs brasileiras pesquisadas em seu trabalho.

\section{O MÉTODO TEMAGUIDE DE GESTÃO DA INOVAÇÃO}

O método proposto pela organização empresarial espanhola Fundación Cotec para la Innovación Tecnológica denominado Technology Management Guide - TEMAGUIDE, traduzido pelo presente estudo como Diretrizes Metodológicas para Gestão de Tecnologia e Inovação Empresarial, “[...] ordena as tarefas e preocupações fundamentais para uma adequada gestão tecnológica dentro das empresas, as explica, indica formas ou procedimentos para levá-las a cabo e as ilustra com exemplos concretos de experiências empresariais [...]" (COTEC, 1999, p. 9). O foco do método TEMAGUIDE está na integração dos típicos processos empresariais (estratégia, operações, finanças, recursos humanos, tecnologia, qualidade e meioambiente) com os processos de inovação, sendo estes o desenvolvimento de novos produtos, a inovação de processos, o desenvolvimento de estratégia tecnológica e a aquisição de tecnologia.

O TEMAGUIDE propõe um marco ou modelo conceitual simples para facilitar a abordagem de gestão da inovação. Ele se baseia em cinco elementos que se assemelham àquilo que uma empresa deve fazer muitas vezes em momentos diferentes e em vários tipos de situações: i) monitoramento [scan], ii) focalização [focus], iii) capacitação [resource], iv) implementação [implement] e v) aprendizado [learning] (COTEC, 1999, p. 14).

Não menos importante, cabe destacar que as cinco etapas ou atividades propostas pelo modelo TEMAGUIDE não são necessariamente sequenciais, podendo ser simultâneas, sendo possível o início do processo de inovação em qualquer uma delas (COTEC, 1999, p. 26). A ênfase que se deve atribuir a cada

\footnotetext{
${ }^{6}$ PERINI, F. A. B. et al. Gestão de tecnologia em grandes e pequenas empresas: uma análise comparativa de ferramentas. In: Simpósio de Gestão da Inovação Tecnológica. 22., Salvador, 2002. Anais... Salvador:PGT/USP.

${ }^{7}$ MORAIS, E. F. C. Inteligência competitiva: estratégias para pequenas empresas. Brasília: GH Comunicação/ Universidade de Brasília, 1999.
} 
elemento ou etapa variará de empresa para empresa e de acordo com cada situação em particular (COTEC, 1999, p. 15).

Figura 1- Modelo conceitual dos elementos-chave da inovação tecnológica

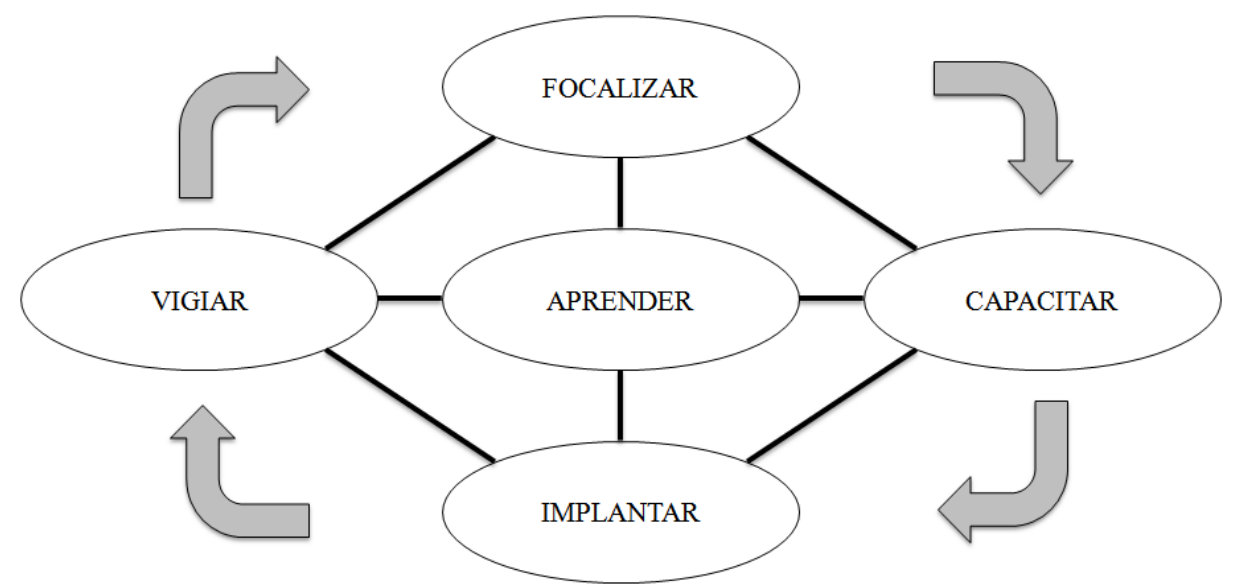

Fonte: COTEC (1999)

A cada uma das cinco etapas ou elementos do modelo, o TEMAGUIDE associa ferramentas e técnicas que podem ser empregadas como apoio conceitual-metodológico para o seu cumprimento e obtenção dos resultados esperados. Segundo Cotec (1998, p. 14),

Os cinco elementos do modelo podem ser apoiados com ferramentas e técnicas, mas isso não é o essencial. O modelo, em qualquer caso, tem um valor intrínseco de reconhecer a posição de uma empresa ou uma equipe em qualquer projeto ou atividade. $\mathrm{O}$ modelo sugere que no desenvolvimento de um projeto ou atividade existe um ciclo iterativo de aprendizagem e não um simples processo em etapas consecutivas.

Assim, a primeira etapa, "monitorar", implica observar o ambiente na busca por sinais sobre a necessidade de inovação e potenciais oportunidades. As ferramentas associadas a esta etapa são: análise de mercado, prospectiva tecnológica, benchmarking, análise de patente, auditorias, criatividade, trabalho em rede, avaliação ambiental. A segunda etapa, "focalizar", significa focar a atenção e os esforços em uma estratégia em particular que traga melhores resultados para o negócio ou a solução para um determinado problema. As ferramentas indicadas para esta fase são: análise de mercado, prospectiva tecnológica, benchmarking, análise de patente, auditorias, gestão de portfólio, avaliação de projetos, criatividade, trabalho em rede, funcionamento em equipe, funcionamento ajustado, análise de valor, avaliação ambiental. Por sua vez, a terceira etapa, "capacitar-se", trata-se de adquirir o conhecimento (explícito e tácito) necessário para desenvolver a estratégia selecionada. São ferramentas indicadas para esta etapa: avaliação de projetos, criatividade, gestão de direitos de propriedade intelectual e industrial, gestão de interfaces, gestão de projetos, trabalho em rede, funcionamento em equipe. A quarta etapa, "implementar", refere-se à execução da alternativa selecionada e as ferramentas auxiliares são: análise de mercado, criatividade, gestão de interface, gestão de projetos, trabalho em rede, funcionamento em equipe, gestão da mudança, funcionamento ajustado, análise de valor, melhoria contínua. A quinta etapa, embora influencie todas as demais, "aprendizado", refere-se ao processo de internalizar conhecimentos das experiências de sucessos e insucessos durante o processo de inovação ou, ainda, aprender com os êxitos ou 
fracassos. As ferramentas indicadas para esse processo são análise de mercado, benchmarking, auditorias, gestão de portfólio, avaliação de projetos, criatividade, trabalho em rede, funcionamento em equipe, funcionamento ajustado, melhoria contínua, avaliação ambiental ( COTEC, 1999, p. 14-17). Embora nesta última etapa o TEMAGUIDE não faça menção explícita à Gestão do Conhecimento, pode-se inferir que esse campo disciplinar poderá contribuir para a com esse processo de aprendizagem organizacional.

Assim, no total o TEMAGUIDE sugere o emprego de 18 (dezoito) ferramentas, que se desdobram, por sua vez, em 71 técnicas específicas, relacionadas às diversas etapas de implementação do modelo, igualmente tributárias de campos disciplinares diversos como gestão da qualidade, gestão de projetos, gestão do conhecimento, planejamento estratégico, gestão de recursos humanos, marketing, dentre outros. No Quadro 1 são relacionadas nominalmente as dezoito ferramentas de apoio à gestão da inovação e respectivas técnicas específicas.

Quadro 1 - Relação de ferramentas de gestão tecnológica e da inovação e técnicas específicas

(Continua)

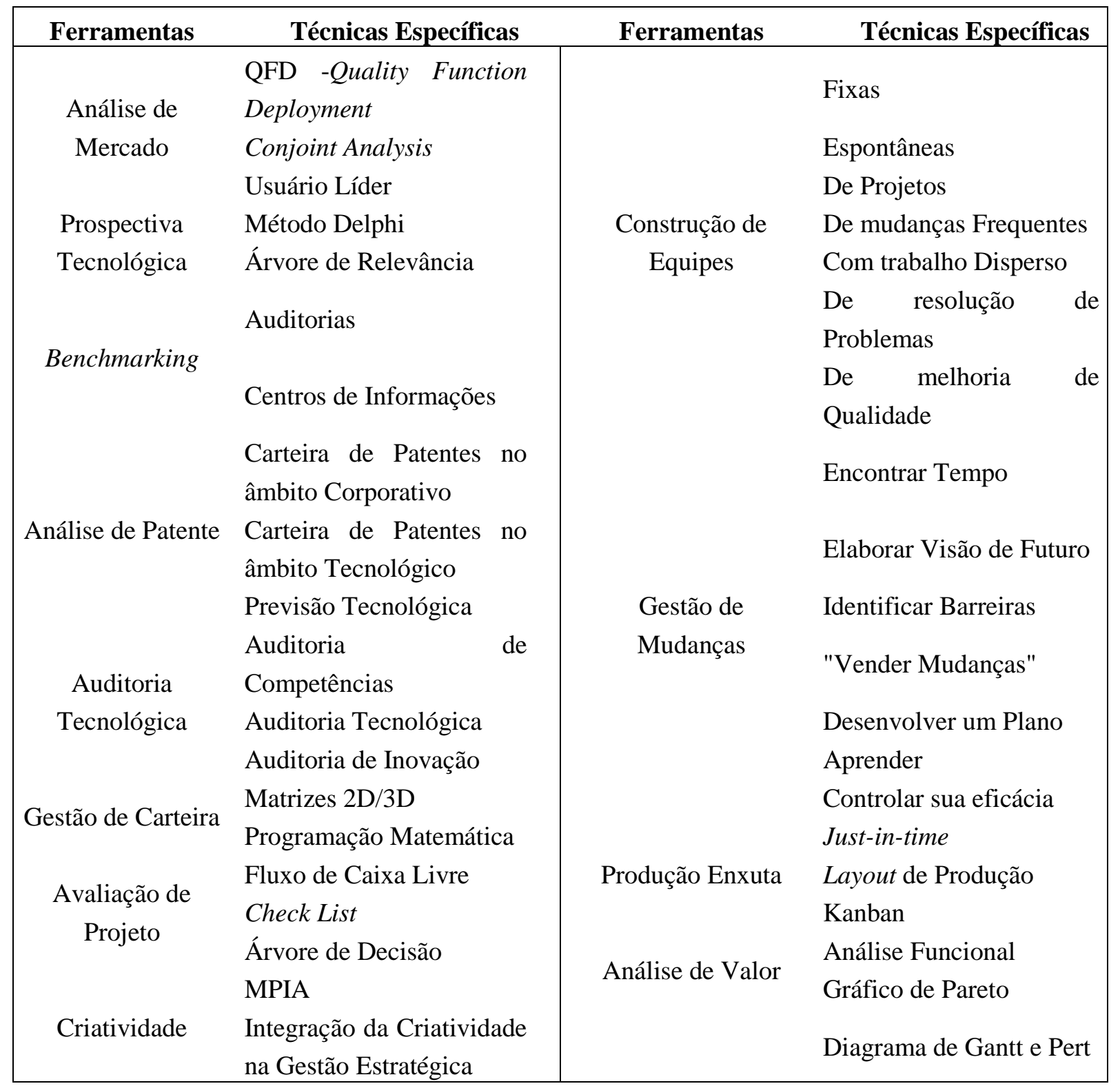


Quadro 1 - Relação de ferramentas de gestão tecnológica e da inovação e técnicas específicas

(Conclusão)

\begin{tabular}{|c|c|c|c|}
\hline Ferramentas & Técnicas Específicas & Ferramentas & Técnicas Específicas \\
\hline $\begin{array}{c}\text { Gestão de } \\
\text { Propriedade } \\
\text { Industrial e } \\
\text { Intelectual }\end{array}$ & Sistemas Patentes & $\begin{array}{l}\text { Melhoria } \\
\text { Contínua }\end{array}$ & Ciclo de Resoluções \\
\hline & Licenciamento & & Brainstorming \\
\hline & Transferências & & $\begin{array}{c}\text { Diagrama de Causa e } \\
\text { Efeito }\end{array}$ \\
\hline & $\begin{array}{l}\text { Contratos de } \\
\text { Consórcios }\end{array}$ & & Listas de Revisão \\
\hline $\begin{array}{l}\text { Gestão de } \\
\text { Interfaces }\end{array}$ & $\begin{array}{c}\text { Estrutural vs de } \\
\text { Processos }\end{array}$ & & Diagramas de Fluxo \\
\hline & Pessoal vs Impessoal & & $\begin{array}{c}\text { Implementação de } \\
\text { Políticas }\end{array}$ \\
\hline Gestão de Projetos & $\begin{array}{c}\text { Desmembramento de } \\
\text { Trabalho }\end{array}$ & Gestão Ambiental & $\begin{array}{c}\text { Minimização de Lixo e } \\
\text { Reciclagem }\end{array}$ \\
\hline & Cronogramas & & $\begin{array}{c}\text { Design Produto } \\
\text { Sustentável } \\
\end{array}$ \\
\hline & Fluxogramas & & Marketing Ambiental \\
\hline & $\begin{array}{c}\text { Método Caminho } \\
\text { Crítico }\end{array}$ & & $\begin{array}{c}\text { Análise do Ciclo de } \\
\text { Vida }\end{array}$ \\
\hline & $\begin{array}{c}\text { Monitoramento de } \\
\text { Marcos }\end{array}$ & & $\begin{array}{c}\text { Ecossistemas } \\
\text { Industriais }\end{array}$ \\
\hline Trabalho em Rede & Longo Prazo & & $\begin{array}{c}\text { Sistemas de Gestão do } \\
\text { Meio-ambiente }\end{array}$ \\
\hline & Curto Prazo & & $\begin{array}{c}\text { Gestão do Meio- } \\
\text { Ambiente na } \\
\text { Qualidade Total }\end{array}$ \\
\hline & $\begin{array}{c}\text { Relacionamentos } \\
\text { Informais }\end{array}$ & & Auditorias Ambientais \\
\hline & Acordos de Relutância & & $\begin{array}{c}\text { Contabilidade total dos } \\
\text { Custos }\end{array}$ \\
\hline & & & $\begin{array}{c}\text { Relatórios da Gestão } \\
\text { do Meio-ambiente }\end{array}$ \\
\hline
\end{tabular}

\section{O SETOR DE EQUIPAMENTOS MÉDICOS, HOSPITALARES E ODONTOLÓGICOS (EMHO)}

O mix produtos ofertados pelas empresas do setor EMHO é bastante amplo. O setor compreende a produção de uma série de produtos e tecnologias que "vão desde os mais tradicionais, como seringas, até equipamentos sofisticados que incorporam tecnologias de informação e comunicação e nanotecnologia" 
(FIESP, 2009). Ainda segundo o estudo conduzido por FIESP (2009), o setor brasileiro de EMHO é composto por 2.139 empresas que empregam 44.385 trabalhadores. A atividade é caracterizada essencialmente pela participação de empresas de micro e pequeno porte. Do total de empresas desse setor, 96,4\% são de micro e pequeno porte (até 99 pessoas ocupadas), 3,2\% de médio porte (de 100 a 499 pessoas ocupadas) e $0,4 \%$ de grande porte (mais de 500 pessoas ocupadas). Em termos de geração de emprego, as micro e pequenas empresas desse setor são responsáveis por 48,8\% dos postos de trabalho, empresas de médio porte por 32,2\% e de grande porte por 19\% (FIESP, 2009). Quanto à origem do capital, as empresas são predominantemente de capital nacional, correspondendo a 79,64\% das empresas (RIEG; ALVES FILHO, 2003, p. 297). As vendas estão distribuídas entre 48\% para o setor privado, $44 \%$ para o governo e $8 \%$ para o mercado externo (IPT, 2008).

Segundo FIESP (2009), o "Estado de São Paulo representa um dos principais territórios produtivos para a cadeia de EMHO no Brasil", se apresentando como o maior estado produtor de EMHO do país. Nele estão localizados $45 \%$ das empresas desse setor, o equivalente a 964 estabelecimentos industriais sendo, 920 micro e pequenas empresas (920), 41 média empresa e 3 grandes empresas. Ainda de acordo com o relatório produzido por FIESP (2009), em termos de trabalhadores empregados, a indústria de EMHO paulista possui em seus estabelecimentos 9.967 postos de trabalho, correspondendo a 22,5\% do pessoal ocupado nessa indústria no Brasil. Do total de trabalhadores desse setor no estado de São Paulo, 58,6\% (5.838) estão na micro e pequena empresa, 31,7\% (3.162) na média empresa e 9,7\% (967) na grande empresa. O município de São Paulo concentra o maior número de empresas produtoras de EMHO no Brasil. A Região Metropolitana de São Paulo, que engloba 39 municípios, corresponde a mais da metade (54,7\%) dos estabelecimentos deste setor no Estado de São Paulo.

Por seu turno, a cidade Ribeirão Preto (SP) responde por 5,1\% (49) do número de estabelecimentos da indústria de EMHO paulista e por 2,3\% dos estabelecimentos da indústria de EMHO brasileira. O Arranjo Produtivo Local (APL) de EMHO de Ribeirão Preto responde também por 5,3\% (1.291) do número de trabalhadores ocupados no setor de EMHO no Estado de São Paulo e por 2,9\% das ocupações na indústria nacional de EMHO (FIESP, 2009). Do total de estabelecimentos desse setor em Ribeirão Preto, $96 \%$ são de micro e pequeno porte (63 estabelecimentos) e o restante de médio porte, $4 \%$ (2 estabelecimentos), sem estabelecimentos com mais de 500 funcionários. No que se refere à distribuição dos trabalhadores entre os portes de empresa desse setor no município: 581 estão ocupados nas micros e pequenas empresas $(45 \%)$ e 710 nas médias empresa (55\%). O rendimento médio do pessoal ocupado nessa indústria local é de $\mathrm{R} \$ 1.294,45$, inferior à média de rendimentos dos empregos ocupados nessa indústria no estado de São Paulo, de R\$1.578,30 (FIESP, 2009).

\subsection{Inovação nas empresas do setor de Emho}

O setor de EMHO tem fundamental importância para o suprimento do mercado de produtos para a saúde. As indústrias que compõem o setor possuem mundialmente um elevado grau de inovação, de conhecimento científico e tecnológico, o que lhes confere dinamismo quanto ao desenvolvimento e aperfeiçoamento de produtos e à competitividade (FIESP, 2009, p. 6). Segundo Furtado e Souza (2000), o desenvolvimento tecnológico na indústria de EMHO apresenta ritmo bastante acelerado, apoiado em múltiplas fontes de insumos. Dinamismo tecnológico do setor EMHO é impulsionado pelos avanços científicos e tecnológicos e pelas inovações de setores como mecânica de precisão, eletrônica digital, informática e química, que fortalecem a oferta da indústria de equipamentos médico-hospitalares. 
Estudo conduzido por IPT (2008), referente ao desenvolvimento tecnológico do setor, aponta que as empresas nacionais de EMHO são mais inovadoras que a média das empresas da indústria de transformação. Do total das empresas, $68 \%$ do setor de EMHO foram consideradas inovadoras, contra 33,5\% da indústria de transformação (IBGE ${ }^{8}, 2003$ apud IPT, 2008, p. 55). A taxa de inovação em produto é de $44,7 \%$ para a indústria de EMHO, enquanto da indústria de transformação é de 33,5\%. Com relação à inovação de processos, ambas apresentam taxa de 30,6\%. Já os gastos com atividades internas de P;D das

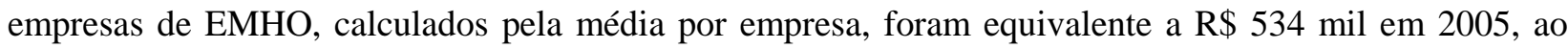
passo que em 2000 tais gastos foram de R $\$ 345$ mil, expressando um crescimento de $54 \%$ no período. "Entre os principais obstáculos à inovação no setor estão os riscos econômicos e os elevados custos associados à inovação" (IPT, 2008).

\section{RESULTADOS}

Na presente seção são apresentados os resultadosobtidos pelo presente trabalho a partir do estudo de caso realizado em uma empresa de pequeno porte do setor de equipamentos médicos, hospitalares e odontológicos situada no município de Ribeirão Preto, Estado de São Pauo (Brasil).

\subsection{Breve caracterização da empresa}

De acordo com a Classificação Nacional de Atividades Econômicas (CNAE) a empresa, objeto da pesquisa de campo, tem como atividade principal a fabricação de materiais para medicina e odontologia (código CNAE 32.50-7-05). A empresa, tem mais de 40 anos de atuação e produz três diferentes linhas de produtos médico-hospitalares. Seus produtos são destinados ao mercado interno e externo, tendo como principais destinos no exterior países da América Latina, Leste Europeu e África. Pela classificação do BNDES sobre o porte de empresas, a companhia estudada é uma pequena empresa, com faturamento entre 2,4 e 16 milhões de reais. A organização possui oito áreas departamentais, sendo que uma delas foi recentemente constituída com o propósito voltado para ações de inovação.A empresa possui um total de 66 funcionários, sendo: 41 empregados alocados na área operacional; quatro (4) exclusivamente no departamento de inovação; outros cinco (5), membros de projetos e gerentes funcionais; e 16 alocados em outras áreas administrativas, como $\mathrm{RH}$, finanças, entre outras. $\mathrm{O}$ total do pessoal alocado na área de inovação representa $13,6 \%$ da dos recurso humanos da empresa.O orçamento anual, médio dos últimos três anos, de projetos em relação a média do faturamento, é 7\%. O orçamento desses projetos é destinado ao desenvolvimento de novos produtos e melhorias de processos. Parte deste orçamento é financiada por recursos de órgãos de fomento, em cerca de 70\%. Apenas pela Financiadora de Estudo e Projetos (FINEP), agência governamental de fomento à inovação, a empresa já teve três projetos contemplados por duas modalidade de apoio: duas de demanda espontânea e umado Programa de Subvenção Econômica à Inovação.

\subsection{Resultados e análise das entrevistas}

De maneira geral, constatou-se que a maior parte das ferramentas de gestão da inovação relacionadas em TEMAGUIDE não é utilizada pela empresa pesquisada. A maioria dos objetivos que justitificariam o uso dessas ferramentas é atingida por meio de outras práticas, ou práticas equivalentes,

${ }^{8}$ IBGE (Instituto Brasileiro de Geografia e Estatística). Pesquisa industrial de inovação tecnológica 2003. Rio de Janeiro: IBGE, 2005. 148 p. Disponível em: 〈http://www.ibge.gov.br>.

Nucleus,v.12,n.2,out.2015 
próprias da empresa. Das 18 ferramentas de gestão da inovação apresentadas em TEMAGUIDE, apenas 8 (ou 44\%) são aplicadaspela empresa. Os resultados encontrados são condizentes com os estudos abordados na seção 3.2 do presente trabalho que revelam a falta de utilização de técnicas e práticas específicas de gestão da inovação por pequenas e médias empresas. Os principais motivos para a não aplicação destas técnicas estão relacionadas a limitações típicas de uma pequena e média empresa (PME), como o desconhecimento sobre as ferramentas de gestão da inovação e percepção reduzida referente a importância da inovação para a competitividade dos negócios.

Como cada ferramenta constante em TEMAGUIDE apresenta um objetivo específico, a pesquisa procurou conhecer outras práticas ou práticas equivalentes realizadas pela empresa que, na percepção do entrevistado, poderiam atender aos mesmos objetivos. Considerando, assim, tais práticas, o entrevistado declarou serem aplicadas 12 ferramentas (ou 67\% do total) semelhantes àquelas presentes em TEMAGUIDE, porém com denominação ou forma de implementação diversa. A utilização de ferramentas equivalentes foi justificada pelo entrevistado devido amaior simplicidade de implementação e aplicação. Foi possível constatar que muitas dessas ferramentas são mais condizentes com a realidade de uma pequena e média empresa, porém algumas vezes é verificado o restrito conhecimento sobre as ferramentas de gestão da inovação, bem como a conscientizaçãode sua utilização por parte da empresa.

Conforme já exposto, cada ferramenta proposta pelo TEMAGUIDE se desdobra em técnicas específicas de aplicação. Assim, no tocante à utilização de técnicas específicas, no âmbito de cada uma das 18 ferramentas, aplicadas e conhecidas pela empresa, bem como a quantidade de técnicas consideradas de interesse para implementação, constatou-se que a maior parte dessas técnicas não é aplicada pela empresa e apenas uma minoria delas é conhecida ou considerada de interesse para serem implementadas. De um total de 71 técnicas específicas, apenas 29 são aplicadas pela empresa, o que representa $41 \%$ do total. Esta proporção é condizente com a proporção de $44 \%$ das ferramentas de gestão da inovaçãonão utilizadas, conforme já mencionado. Considerando apenas as técnicas específicas que não são aplicadas pela empresa - ou seja, 42 técnicas - apenas 15 delas eram conhecidas anteriormente pela empresa, o representa 36\%. Esta constatação também é condizente a falta de conhecimento e divulgação de informações sobre ferramentas de gestão da inovação, conforme é apontado pelos estudos apresentados na seção 3.2 do presente trabalho.

Após a apresentação e esclarecimentos à empresa sobre as técnicas específicas relacionadas pelo TEMAGUIDE, questionou-se o entrevistado sobre o interesse da empresa em implementar estas técnicas, até então desconhecidas pela empresa. Das 42 técnicas específicas não aplicadas, somente 14 foram apontadas como sendo de interesse da empresa, o representa 33\%.

O entrevistado faz referência ao uso de outras práticas, próprias da empresa, as quais são consideradas mais simples e satisfatórias para atenderem as atuais necessidades, como sendo o motivo principal para não implementar as técnicas específicas apresentadas em TEMAGUDE. Além disso, a aplicação de algumas técnicas específicas foi considerada fora da realidade de uma pequena empresa. No Quadro 2 são apresentadas de forma resumida as informações, coletadas durante as entrevistas, sobre a aplicação das ferramentas de gestão tecnológica e respectivas técnicas específicas pela empresa pesquisada. 
Quadro 2 - Aplicação das ferramentas de gestão da inovação e respectivas técnicas específicas

(Continua)

\begin{tabular}{|c|c|c|}
\hline $\begin{array}{c}\text { Ferramentas } \\
\text { de Gestão da Inovação }\end{array}$ & $\begin{array}{l}\text { Técnicas Específicas } \\
\text { TEMAGUIDE }\end{array}$ & $\begin{array}{c}\text { Uso de práticas equivalentes pela } \\
\text { empresa }\end{array}$ \\
\hline Análise de Mercado & - & $\begin{array}{l}\text { Reuniões com a assistência técnica; } \\
\text { Análise dados do SAC; } \\
\text { Questionários com representantes de } \\
\text { vendas; e reuniões com grandes } \\
\text { clientes }\end{array}$ \\
\hline $\begin{array}{l}\text { Prospectiva } \\
\text { Tecnológica }\end{array}$ & - & - \\
\hline Benchmarking & - & $\begin{array}{l}\text { Participação em feiras nacionais e } \\
\text { internacionais; acompanhamento de } \\
\text { editais públicos nacionais e } \\
\text { internacionais; Reuniões com } \\
\text { pessoal de vendas e da assistência } \\
\text { técnica }\end{array}$ \\
\hline Análise de Patente & - & $\begin{array}{l}\text { Monitoramento de patentes realizado } \\
\text { por Terceiros para análise qualitativa } \\
\text { de tendências }\end{array}$ \\
\hline $\begin{array}{l}\text { Auditoria } \\
\text { Tecnológica }\end{array}$ & - & - \\
\hline Gestão de Carteira & - & $\begin{array}{l}\text { Os projetos são condicionados aos } \\
\text { Editais da FINEP }\end{array}$ \\
\hline Avaliação de Projeto & Fluxo de Caixa Livre & - \\
\hline Criatividade & - & $\begin{array}{l}\text { Reuniões e práticas de } \\
\text { Brainstorming }\end{array}$ \\
\hline Gestão de PII & - & - \\
\hline \multirow{2}{*}{ Gestão de Interfaces } & Orientada para Estrutura & \multirow{2}{*}{$\begin{array}{l}\text { Reunião para gerenciar o Balanced } \\
\text { Scorecard (BSC) }\end{array}$} \\
\hline & Orientada para Processos & \\
\hline \multirow{4}{*}{ Gestão de Projetos } & Desmembramento de Trabalho & \multirow{4}{*}{ Prestação de contas à FINEP } \\
\hline & Cronogramas & \\
\hline & Fluxogramas & \\
\hline & Monitoramento de Marcos & \\
\hline \multirow{2}{*}{ Trabalho em Rede } & De Curto Prazo & \multirow{2}{*}{-} \\
\hline & Relacionamentos Informais & \\
\hline \multirow{5}{*}{$\begin{array}{l}\text { Construção de } \\
\text { Equipes }\end{array}$} & Fixas & \multirow{4}{*}{-} \\
\hline & Espontâneas & \\
\hline & De Projetos & \\
\hline & De resolução de Problemas & \\
\hline & De melhoria de Qualidade & \\
\hline
\end{tabular}

Nucleus,v.12,n.2,out.2015 
Quadro 2 - Aplicação das ferramentas de gestão da inovação e respectivas técnicas específicas

(Conclusão)

\begin{tabular}{|c|c|c|}
\hline \multirow{5}{*}{$\begin{array}{l}\text { Gestão de } \\
\text { Mudanças }\end{array}$} & Encontrar Tempo & \multirow{5}{*}{ Força da decisão da alta diretoria } \\
\hline & Elaborar Visão de Futuro & \\
\hline & Identificar Barreiras & \\
\hline & "Vender Mudanças" & \\
\hline & Controlar sua eficácia & \\
\hline Produção Enxuta & - & $\begin{array}{l}\text { BSC; Prazos de Entrega; Índice } \\
\text { Vendas vs. Produção; Índice de } \\
\text { Ociosidade; Formulação de Estoque; } \\
\text { e Painel de Desempenho. }\end{array}$ \\
\hline Análise de Valor & - & $\begin{array}{l}\text { Reunião multidisciplinar para análise } \\
\text { qualitativa }\end{array}$ \\
\hline \multirow{6}{*}{ Melhoria Contínua } & Ciclo de Resoluções & \multirow{6}{*}{$\begin{array}{l}\text { Atualizações de certificados: CE, } \\
\text { ISO 13450; Certificado Boas } \\
\text { Práticas de Fabricação; e Inmetro }\end{array}$} \\
\hline & Brainstorming & \\
\hline & Diagrama de Causa e Efeito & \\
\hline & Listas de Revisão & \\
\hline & Diagramas de Fluxo & \\
\hline & Implementação de Políticas & \\
\hline \multirow{4}{*}{ Gestão Ambiental } & \begin{tabular}{|l} 
Minimização de \\
Reciclagem
\end{tabular} & \multirow{4}{*}{$\begin{array}{l}\text { Auditorias externas de fiscalização } \\
\text { CETESB, Prefeitura, BPF e ISO }\end{array}$} \\
\hline & Seis Sigma & \\
\hline & Qualidade Total & \\
\hline & Auditorias Ambientais & \\
\hline
\end{tabular}

Como pode ser observado, três das ferramentas de gestão da inovação não são utilizadas pela empresa, quer seja segundo a proposição original do TEMAGUIDE, quer seja por meio de práticas equivalentes. São elas: i) Previsão Tecnológica; ii) Gestão de Propriedade Intelectual e Industrial; iii) Auditoria Tecnológica. Apenas para esta última a empresa demonstrou interesse em implementar, sendo que não era previamente conhecida pela mesma. Esta constatação evidencia a carência de divulgação adequada sobre ferramentas de gestão da inovação para que elas sejam aplicadas nas PME. Em relação às ferramentas Prospecção Tecnológica e Gestão de Propriedade Intelectual e Industrial, a principal razão apontada pelo entrevistado para explicar a não aplicação das mesmas está associada à baixa de competitividade do setor. O entrevistado afirma que há menos de cinco concorrentes diretos das principais linhas de produtos da empresa no Brasil. A alta regulamentação do setor é indicada pelo entrevistado como a principal barreira que desestimula a entrada de novos concorrentes, inclusive estrangeiros. As análises do setor de EMHO apontam para outra direção em relação à competitividade do setor, as quais indicam forte competição. Este fato oferece risco tanto às exportações brasileiras quanto à participação no mercado doméstico, se considerado a possibilidade de entrada de players globais, como a China, e/ou a importação dos produtos destes. Geralmente em pequenas e médias empresas, a conscientização sobre as ameaças competitivas se baseia em visões de curto e médio prazo. 
O entrevistado afirmou ainda que a demanda por inovação do mercado doméstico é relativamente baixa e o pequeno porte das empresas atuantes no setor nacional são fatores adicionais que não justificam a aplicação das ferramentas Prospecção Tecnológica e Gestão de Propriedade Intelectual e Industrial. Vale a pena mencionar que esta última ferramenta não é utilizada pela empresa em virtude da ausência de patentes. No entanto, as justificativas apontadas para a empresa não possuir patentes são as mesmas.

As motivações apontadas pelo entrevistado também estão apoiadas por uma visão mais imediatista por parte da empresa. Se levado em consideração a atual dinâmica de mudanças das preferências e dos comportamentos dos consumidores, a empresa pode se deparar com necessidades ou exigências repentinas do mercado. Este fato pode ocorrer inclusive com o assédio ou entrada de novos produtos.

A principal dificuldade relacionada às condições de uma pequena empresa, apontada pelo entrevistado, diz respeito ao custo relativamente alto de implementação das técnicas ou alocação de recursos escassos para suas aplicações. Como por exemplo, nos casos mencionados das técnicas Método Delphi (Prospecção Tecnológica) e Usuário Líder (Analise de Mercado). Neste caso, entende-se que esta situação é comum à maioria das PMEs brasileiras e sua superação necessita de apoio de políticas públicas ou ações conjuntas entre empresas.

Como pôde ser visto também no Quadro 2 parte das ferramentas de gestão da inovação sugeridas em TEMAGUIDE é suprida, exclusivamente, por outras práticas, ou práticas equivalentes, próprias da empresa. São 7 no total: Análise de Mercado; Benchmarking; Análise de Patentes; Gestão de Carteira; Criatividade; Produção Enxuta; e Análise de Valor. Percebe-se que a maioria dessas práticas é condizente com as condições de uma PME como, por exemplo, Produção Enxuta e Análise de Valor. O sistema denominado de Produção Enxuta tem como princípios básicos o comprometimento da organização com a redução de desperdícios e a eliminação de estoques. Com efeito, as práticas apontadas pela empresa são apenas um esforço inicial para tentar alcançar tal compromisso em toda organização, mas não configuram uma gestão de Produção Enxuta, como tal. Com relação à ferramenta Analise de Valor, a realização de reuniões com equipe multidisciplinar não atribui valor quantitativo aos retornos de componentes funcionais ou às melhorias decididas. Os resultados dessas reuniões são referentes à priorização ou não de melhorias e incorporação de componentes funcionais no processo ou produto a ser desenvolvido.

No Quadro 2 são apresentadas também as oito ferramentas de gestão da inovação que são utilizadas por meio da aplicação de pelo menos uma das técnicas específicas presentes em TEMAGUIDE: Avaliação de Projetos; Gestão de Interfaces; Gestão de Projetos; Trabalho em Rede; Construção de Equipes; Gestão de Mudanças; Melhoria Contínua; e Gestão Ambiental. Depreende-se que a utilização dessas ferramentas é decorrente da aplicação das técnicas específicas mais simples ou que fazem parte de programas de melhoria de qualidade. Outras ainda, são técnicas exigidas por órgãos de fomento ou requeridas na fiscalização de órgão públicos ou instituições de certificações.

Algumas das técnicas específicas são aplicadas para atender objetivos distintos do proposto pelo TEMAGUIDE, como é o caso, por exemplo, de Gestão de Interfaces. Apesar das técnicas e práticas apontadas pela empresa apoiarem paralela ou indiretamente o objetivo desta ferramenta, suas aplicações não têm como propósito principal gerenciar relacionamentos entre as interfaces internas ou externas da empresa.

No Quadro 3 são apresentadas as técnicas específicas apresentadas em TEMAGUIDE que a empresa tem interesse na aplicação, inclusive com os respectivos prazos para empresa iniciar o processo de implementação.

Nucleus,v.12,n.2,out.2015 
Quadro 3 - Interesse de implementação de técnicas específicas

\begin{tabular}{|c|c|c|c|}
\hline $\begin{array}{c}\text { Ferramentas } \\
\text { de Gestão da } \\
\text { Inovação }\end{array}$ & $\begin{array}{c}\text { Técnicas específicas } \\
\text { a serem implementadas }\end{array}$ & $\begin{array}{l}\text { Até } 6 \\
\text { meses }\end{array}$ & $\begin{array}{c}\text { De } 12 \text { a18 } \\
\text { meses }\end{array}$ \\
\hline \multirow{2}{*}{ Análise de Mercado } & QFD & • & \\
\hline & Conjoint Analysis & • & \\
\hline \multirow[t]{2}{*}{ Análise de Patente } & Previsão Tecnológica & • & \\
\hline & Aud. de Competências & & $\bullet$ \\
\hline \multirow[t]{2}{*}{ AuditoriaTecnológica } & Aud. de Tecnologias & & - \\
\hline & Aud. de Inovação & & $\bullet$ \\
\hline Criatividade & MPIA & • & \\
\hline Gestão de Mudança & Aprender & • & \\
\hline \multirow{2}{*}{ Análise de Valor } & Análise Funcional & $\bullet$ & \\
\hline & Gráfico de Pareto & - & \\
\hline \multirow{4}{*}{ Gestão Ambiental } & Design Produto & • & \\
\hline & Marketing Ambiental & • & \\
\hline & Anál. do Ciclo de Vida & • & \\
\hline & Relatórios MA & - & \\
\hline
\end{tabular}

O interesse da empresa pelas técnicas específicas constantes no Quadro 3 evidencia o potencial existente de aplicação ferramentas de gestão da inovação em empresas de pequeno e médio porte, após a simples difusão de informações acerca de seus objetivos, aplicação e resultados a serem obtidos. A intenção de se iniciar o processo de implementação dessas técnicas no curto prazo - entre 6 e 18 meses aponta também, de certa forma, a viabilidade técnica e organizacional existente na empresa para tanto.

Por fim, com relação às técnicas específicas não consideradas de interesse pela empresa para serem implementadas, o entrevistado apontou as seguintes justificativas: complexidade da técnica versus benefícios resultantes; baixo número de projetos; cultura tradicional da empresa; ausência de circunstâncias específicas; alto custo de implantação; as mudanças são desestimuladas pela forte regulamentação do setor ou às exigências de editais de fornecimento/fomento; e outros fatores já mencionados no trabalho.

\section{CONSIDERAÇÕES FINAIS}

A revisão bibliográfica sobre a gestão da inovação em empresas de pequeno porte no Brasil permitiu verificar que as pequenas empresas não fazem uso extensivo de ferramentas de gestão da inovação. A falta de conhecimento dessas técnicas é apontada em alguns estudos como a principal razão para não aplicar essas ferramentas.

O presente trabalho optou por um estudo de caso, de caráter exploratório, para verificar se uma pequena empresa, de um setor intensivo de inovação, aplica ou não as ferramentas e técnicas específicas propostas pelo método TEMAGUIDE (COTEC, 1999). Se não as utiliza, o porquê e, sendo uma empresa caracterizada como inovadora, quais são as práticas utilizadas para realizar sua gestão da inovação. 
Os resultados da pesquisa demonstraram que o nível de utilização das ferramentas e técnicas específicas pela empresa analisada não é diferente do conjunto de empresas pesquisadas em estudos anteriores, ratificando, assim, esta característica de pequena empresa. Após a apresentação de todas as técnicas específicas para o conhecimento da organização, o entrevistado demonstrou interesse em implementar algumas delas, inclusive no curto prazo (em até seis meses). Das 42 técnicas não aplicadas pela empresa, somente 14 foram consideradas para serem implementadas, correspondendo a 33\%. Parcela significativa de 39,4\% do total das 71 técnicas específicas não é considerada interessante para ser aplicada. Caso essas venham a ser implementadas, somadas as 29 técnicas já aplicadas pela empresa, esta contará no futuro com a aplicação de 43 técnicas específicas, correspondendo a 60,4\%. Este fato revela condições favoráveis para aplicação da maior parte das técnicas propostas por TEMAGUIDE.

Com relação às barreiras impostas a aplicação das ferramentas de gestão da inovação, foi verificado que a desconhecimento e falta de informações sobre estas ferramentas não é única dificuldade encontrada pelas pequenas e médias empresas. É necessário também estimular a conscientização da importância da inovação para a competitividade. Dessa forma, políticas públicas devem não apenas difundir adequadamente informações sobre a importância da inovação para a competitividade dos negócios, mas avançar mais decisivamente no oferecimento de programas de capacitação das empresas em métodos, ferramentas e técnicas específicas de gestão da inovação.

Já com relação ao alto custo de aplicação de algumas técnicas específicas - como, por exemplo, o Método Delphi que se a apresenta inviável para uma pequena e média empresa isoladamente, estas podem ser promovidas por várias empresas em pareceria ou financiadas por instituições públicas ou associações empresariais com o intuito de compartilhar os benefícios gerados para o setor como um todo. Estas medidas podem contribuir para assegurar a competitividade dessas empresas frente a forte concorrência internacional presente cada vez mais no mercado doméstico.

Portanto, apesar de algumas restrições típicas de uma pequena empresa, o estudo de caso evidencia o grande potencial existente para aplicação de métodos, ferramentas e técnicas de gestão da inovação no contexto organizacional de uma empresa de pequeno porte, de um setor intensivo em tecnologia e inovação. E o aumento do número de ferramentas e técnicas utilizadas pode significar o aumento da capacidade tecnológica e de inovação e, consequentemente, da competitividade das empresas.

\section{REFERÊNCIAS}

CORAL, E.; OGLIARI, A.; DE ABREU, A. F (Org.). Gestão integrada da inovação: estratégia, organização e desenvolvimento de produtos. São Paulo: Atlas, 2008. 269 p.

COTEC -Fundación para la Innovación Tecnológica. Pautas metodológicas en gestión de la tecnologia y de la innovación para empresas. Tomo 1. Módulo I. "Introducción, Presentación, CD y Módulo I: perspectiva empresarial” - TEMAGUIDE. 1998. $60 \mathrm{p}$.

FAYET, E. A. Inovação: conceitos e teorias. In: FAYET, E. A. (Org.). Gerenciar a inovação: um desafio para as empresas. Curitiba: IEL(PR), 2010. p. 37-51.

FIESP (Federação das Indústrias do Estado de São Paulo). Análise setorial de mercado: setor de equipamentos médicos, hospitalares e odontológicos. São Paulo: FIESP. 47 p.

FUCK; M. P.; VILHA, A. M. Inovação Tecnológica: da definição à ação. Revista Contemporâneos, São Paulo, n. 9, p. 1-21. 2011. 
FURTADO, A. T.; SOUZA, J. H. Setor de equipamentos médicos - evolução do setor de insumos e equipamentos médico-hospitalares, laboratoriais e odontológicos brasileiro, 2000.

GAVIRA, M. O. et al. Gestão da Inovação Tecnológica: uma análise da aplicação do funil de inovação em uma organização de bens de consumo. Revista de Administração Mackenzie, vol. 8, n.1. 2007, p. 77107.

GIL, A. C. Como elaborar projetos de pesquisa. 4. ed. São Paulo: Atlas, 2002. 175p.

IPT (Instituto de Pesquisas Tecnológias do Estado de São Paulo). Competitividade da Indústria Paulista. Propostas de Politicas. São Paulo: IPT. 2008. 133 p.

LAKATOS, E. M; MARCONI, M. A. Fundamentos de metodologia científica. 5. ed. São Paulo: Atlas, 2003. $311 \mathrm{p}$

LIMAS, C. E. A.; SCANDELARI, L.; FRANCISCO, A. C. D. O uso das ferramentas da tecnologia nas pequenas e microempresas de Ponta Grossa (PR). In: ENCONTRO DE ENGENHARIA E

TECNOLOGIA DOS CAMPOS GERAIS, 2., 2008, Campos Gerais. Anais...Campos Gerais: AEAPG, 2008. 8 p.

MAXIMIANO, A. C. A. Teoria geral da administração: da revolução urbana à revolução digital. São Paulo: Atlas, 2006. 504 p.

MILESKI JR., A. Práticas de apoio inseridas em processos de gestão da tecnologia e inovação: um estudo na indústria paranaense. 2008. 111 f. Dissertação (Mestrado em Tecnologia) - Universidade Tecnológica Federal do Paraná, Programa de Pós-Graduação em Tecnologia, Área de Concentração: Tecnologia e Desenvolvimento, Curitiba, 2008.

NATUME, R. Y.; CARVALHO, H. G.; FRANCISCO, A. C. O uso de práticas de gestão de tecnologia e inovação em uma empresa de médio porte do Estado do Paraná. Revista Eletrônica Internacional de Economia da Informação, da Comunicação e da Cultura. v. X, n. 1, 2008. p. 179-202.

PALADINO, G. Introdução. In: FAYET, E. A. (Org.). Gerenciar a inovação: um desafio para as empresas. Curitiba: IEL (PR), 2010. p. 11-16.

RIEG, D. L.; ALVES FILHO, A.G. Esforço tecnológico e desempenho inovador das empresas do setor médico-hospitalar localizadas em São Carlos (SP). Gestão; Produção, v.10, n.3, p.293-310, 2003.

SCHERER, F. O.; CARLOMAGNO, M. S. Gestão da inovação na prática: como ampliar conceitos e ferramentas para alavancar a inovação. São Paulo: Atlas, 2009. 150 p.

SOUZA, D. L. O. D. Ferramentas de gestão de tecnológica: um diagnóstico de utilização nas pequenas e médias empresas industriais da região de Curitiba. 2003. 120 f. Dissertação (Mestrado em Tecnologia) Programa de Pós-Graduação em Tecnologia, Centro Federal de Educação Tecnológica do Paraná, Curitiba, 2003.

TIDD, J.; BESSANT, J.; PAVITT, K. Gestão da Inovação. 3. ed. Porto Alegre: Bookman, 2008. 600 p.

VILHA, A. O. M. Gestão da inovação na indústria brasileira de higiene pessoal, perfumaria e cosméticos: uma análise sob a perspectiva do desenvolvimento sustentável. 2009. $161 \mathrm{f}$. Tese (Doutorado em Política Científica e Tecnológica) - Instituto de Geociências, Universidade Estadual de Campinas, Campinas, 2009. 
